\title{
Channel Matrix Characterization in MIMO Scenario Through Impedance Modulation
}

\author{
B. Monsalve ${ }^{*(1)}, \mathrm{J}_{\text {. Romeu }}{ }^{(1)}$, and S. Blanch ${ }^{(1)}$ \\ (1) Antennalab, Universitat Politècnica de Catalunya, Barcelona, Spain \\ E-mail: bmonsalve@tsc.upc.edu
}

\begin{abstract}
Introduction
The increasing demands on multimedia services require high capacity communication systems jointly with a compact and miniaturized antenna design. In this framework compact MIMO radio systems provide high channel capacity with small size. To characterize the maximum channel capacity the determination of the channel matrix is needed. To do that with a mobile, compact and small RF systems in a traditional way a Network Analyzer and long cables are used which complicate the measurement. In some cases this additional equipment modifies the measurement scenario. To avoid this inconvenience a new methodology to obtain the characteristic channel matrix of a MIMO radio system is presented based on the Radar Cross Section (RCS) measurement method using impedance modulation.

Radar Cross Section measurements are well-known to characterize antennas as a measurement setup [1] and together with impedance modulation allow achieving a small and autonomous device. Using the scattered fields not only the reconstruction of the characteristic parameters or a near-field map [2] of any antenna can be found but also the transmission parameters between antennas. All these properties have been exploited in [3], where a small device was designed, constructed and validated for one port small antennas characterization. Based on this idea a new device has been constructed to characterize two port small antennas.

In this paper a new measurement setup to obtain the characteristic channel matrix of a MIMO radio system is presented using a small and non intrusive device able to characterize two antennas and therefore obtaining the characteristic channel matrix of a MIMO radio system of $\mathrm{Nx} 2$ antennas without using a receiver for each port. The extension to a system of more than two output elements can be easily achieved increasing the complexity of the switching device. To validate the new methodology presented here a 2x2 MIMO channel matrix has been characterized comparing the singular value distribution obtained with a traditional measurement procedure.
\end{abstract}

\section{Formulation}

Assuming the $2 \times 2$ MIMO radio system depicted in Fig. 1(a) the resulting scattering matrix $[\mathrm{S}]^{\mathrm{T}}$ can be considered as a four port network with the equivalent signal flow graph showed in Fig. 1(b). If port 3 and port 4 are loaded with a certain reflection coefficient $\Gamma_{\mathrm{Li}}$, the reflection coefficient at ports 1 and $2\left(\Gamma_{\mathrm{i}=1,2}\right)$ are related to every single value of the scattering matrix $[\mathrm{S}]^{\mathrm{T}}$ and therefore of the channel matrix.

$\mathrm{S}_{11}, \mathrm{~S}_{22}, \mathrm{~S}_{33}$ and $\mathrm{S}_{44}$ are the return losses of transmitting and receiving antennas, respectively. $S_{31}, S_{13}, S_{41}, S_{14}, S_{32}, S_{23}, S_{42}$ and $S_{24}$ are dependent on the gain of each antenna, the polarization coefficient and the distance between the antennas and are directly the coefficients of the channel matrix. $S_{34}$ and $S_{43}$ are the isolation between the two receiving antennas. To obtain the transmission parameters related with the channel matrix aforementioned a reflection measurement has to be done for each transmitting port 
$\left(\Gamma_{\mathrm{i}=1,2}\right)$. From the initial 16 unknowns of the scattering matrix $[\mathrm{S}]^{\mathrm{T}}$ only 8 have to be solved considering reciprocity and also because isolation between ports it is not necessary to reconstruct in order to obtain the channel matrix.

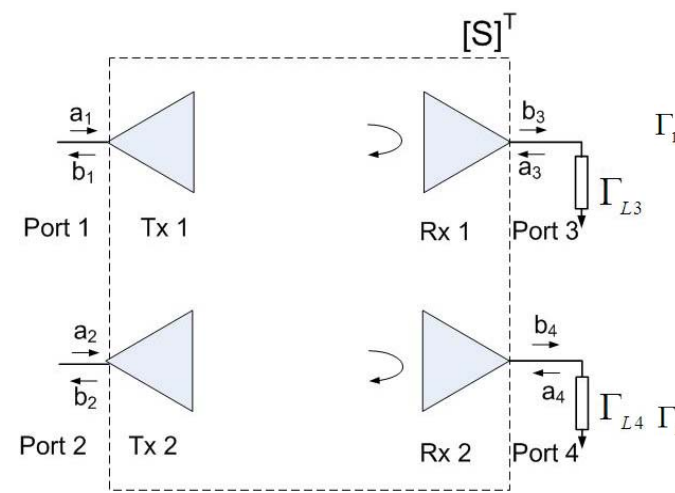

a)

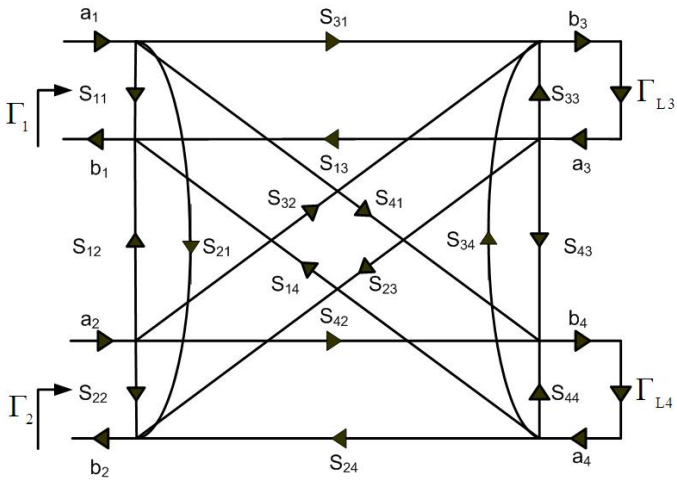

b)

Fig.1 (a) Scattering parameter representation and (b) equivalent signal flow graph.

Just as eight unknowns have to be solved an $8 \times 8$ equation system is needed to characterize the scattering matrix. Changing the reflection coefficient of each receiving port between three known states (short circuit, open circuit and matched load) and combining with a certain methodology the resulting re-radiated field has nine different states. To solve the unknowns only eight states are needed to characterized the scattering matrix.

Once the scattering matrix is determined the channel matrix can be obtained straightforward and after that the singular values are found as follows: first, the channel matrix has been normalized by the Frobenious normalization described in [4] and then, the correlation matrix has been calculated as:

$$
R=H H^{H}
$$

The singular value distribution is obtained from the correlation matrix.

\section{Experimental setup}

Switching device: To produce an impedance modulation at a certain frequency, a change between different load impedances from each port is needed and also the generation of the required frequency modulation. Three different states are required to load each port: an ideal short circuit, open circuit and $50 \Omega$ load have been chosen as load impedances to cover as much different values as the system can. Finally, the important point is a well characterization of the load impedances over the frequency range and achieves different enough load impedances to obtain a better conditioned equation system.

The switching device comprised two SP3T switches working from DC to $3.5 \mathrm{GHz}$ each one of them able to change the load impedance between three different states, a microprocessor to control the load variation of each port with the required frequency and six load impedances (two $50 \Omega$ loads, two short circuits and two open circuits). The microprocessor is changing its output bits of control in order to select the nine different combinations of loads, controlled by the two IP3T switches, between the two ports (three different loads for each port) in a cyclical way with a certain frequency. A complete scheme of the device is shown in Fig. 2 (left).

Measurement setup: The measurement setup is depicted in Fig. 2 (right) and is composed of a system of $2 \times 2$ antennas with the transmitting antennas connected to the measurement 
equipment, and the receiving antennas connected to the communication device described in previous section. As measurement equipment an Agilent Network Analyzer E8236 is used in time domain and is monitored by a laptop. The measurement is done inside an anechoic chamber using an electronic calibration procedure to remove the cable contribution from the transmitting antennas. The input reflection coefficient for each switch position over the frequency range of measurement has to be characterized in order to reconstruct the channel matrix. No external trigger is needed from the communication device to the VNA.

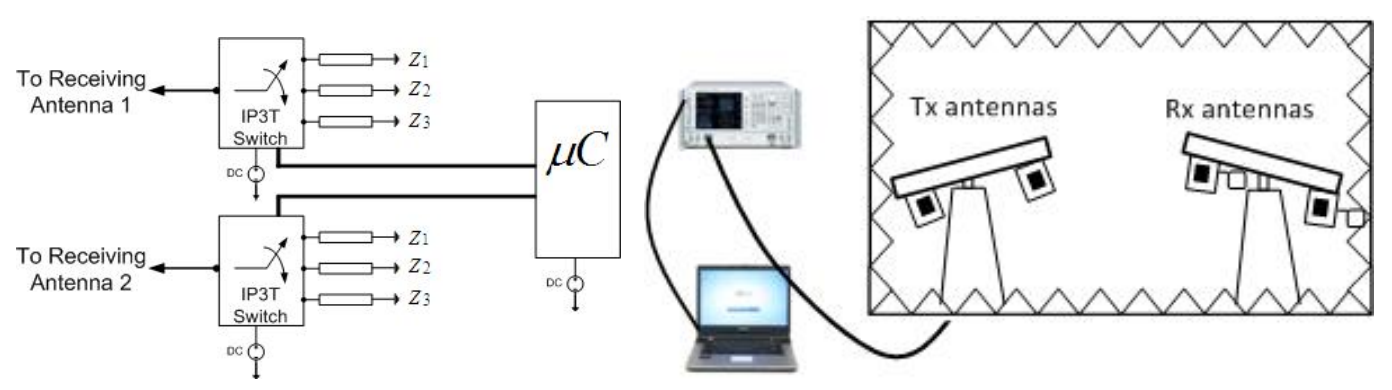

Fig.2 Communication device scheme (left) and measurement setup (right).

\section{Results}

Assuming the measurement setup presented in the previous section, a 2x2 MIMO radio system is characterized for two different receiving antenna distances $(0.7 \lambda$ and $1.2 \lambda)$. Two patch antennas have been used as receiving antennas working at $900 \mathrm{MHz}$ and two ridge horn antennas working between $0.8-3 \mathrm{GHz}$ as transmitting antennas. Distance between the transmitting and receiving antennas was $2.5 \mathrm{~m}$. The results obtained are depicted in Figure 3 where a concordance between the measurements done with the Network Analyzer connected to each port (line traces) and the measurement using the device (asterisk traces) has been observed, validating the measurement setup proposed in this contribution. It can be seen that as longer the distance between receiving antennas nearest the singular values are to each other indicating a higher channel capacity for a longer distance separation.
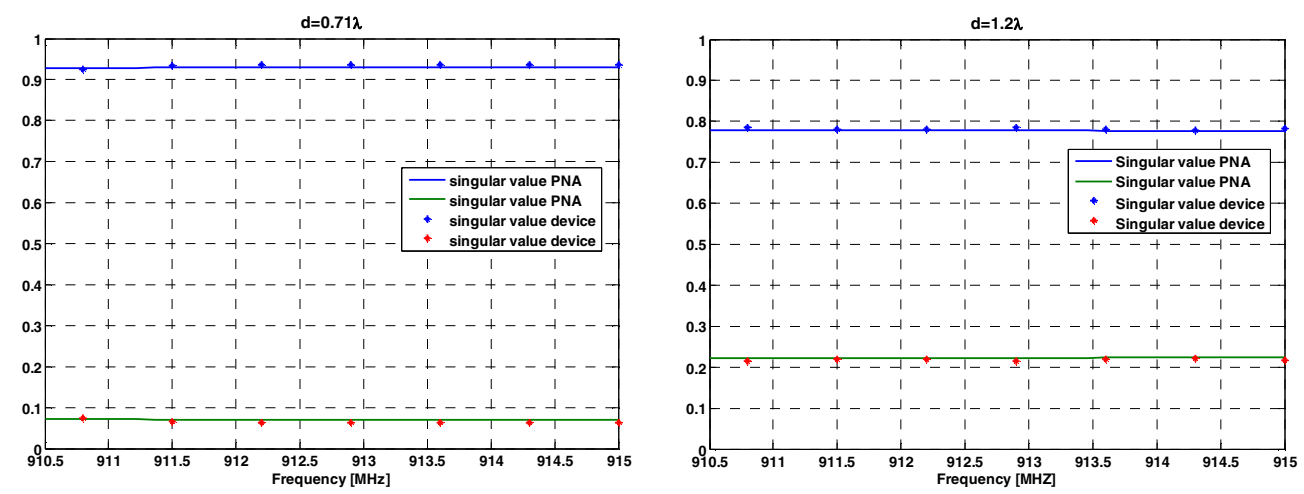

Fig.3 Singular value distribution for different distances between receiving antennas. 


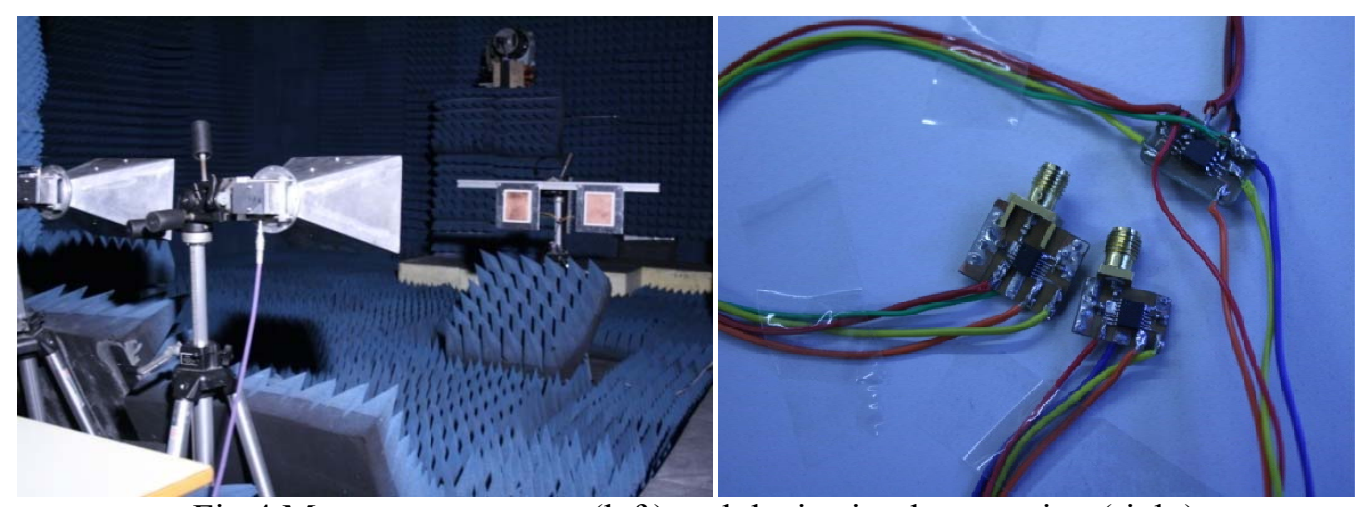

Fig.4 Measurement setup (left) and device implementation (right).

\section{Conclusions}

A new measurement setup and methodology have been proposed to evaluate the singular value distribution for a $2 \times 2$ MIMO radio system based on the use of a small device in the receiving antennas. Therefore, instead of use a receiver for each port only two receivers have to be used in transmitting antennas. Measurements of the singular value distribution using this new measurement setup are in concordance with the traditional measurement procedure validating the methodology presented. Two different receiving antenna distances have been measured and the singular value distribution has been retrieved obtained the expected results.

\section{References}

[1] W. Wiesbeck and E. Heidrich, "Wide-Band Multiport Antenna Characterization by polarimetric RCS Measurements”, IEEE Transactions on Antennas and Propagation, vol.46, nº3, March 1998.

[2] J.C. Bolomey, "Engineering Applications of the Modulated Scatterer Technique".

[3] B. Monsalve, S. Blanch, J. Romeu and Ll. Jofre, "A contactless small antenna characterization through impedance modulation". European Conference on Antennas and Propagation EUCAP 2009, Berlin, 23-27 March 2009, pp. 696-698.

[4] F. De Flaviis, L.Jofre, J.Romeu, and A.Grau, "Multi-Antenna Systems for MIMO Communications", Ed. Morgan \& ClayPool, 2008. 